\title{
Esperança de vida e sua relação com indicadores de longevidade: um estudo demográfico para o Brasil, 1980-2050
}

\author{
Francismara Fernandes Guerra* \\ Moema Bueno Gonçalves Fígoli ${ }^{\star \star}$
}

Quando a mortalidade encontra-se em constante mudança, a tabela de sobrevivência de período não descreve a verdadeira experiência de vida das coortes presentes na população. Nesse sentido, o emprego das taxas correntes de mortalidade na estimativa do tempo médio de vida distorce o conceito original de esperança de vida. Como uma medida de coorte, a longevidade deveria ser estimada para um grupo real de pessoas, mas usualmente refere-se a uma coorte hipotética. Dadas as quedas passadas da mortalidade e as prováveis reduções futuras, a esperança de vida de coorte se torna maior do que a de período. Apesar disso, esta última pode ser vista como um indicador defasado do tempo médio de vida de alguma coorte passada (coorte equivalente). Este artigo explora o tempo requerido para encontrar essa coorte passada (defasagem) e as diferenças entre as esperanças de vida em um dado ano (diferencial) a fim de determinar a translação da medida de período, na perspectiva de coorte. Com a tendência de ganhos da longevidade cada vez menores no Brasil, os resultados indicam que, tanto para homens quanto para mulheres, o diferencial se reduz com o tempo, ao passo que a defasagem torna-se crescente. Isto é, com o passar dos anos, as estimativas correntes de período e coorte tendem a se aproximar, enquanto as coortes equivalentes se tornam mais remotas.

Palavras-chave: Medidas de longevidade. Translação da esperança de vida. Diferencial. Defasagem.

\footnotetext{
* Departamento de Demografia da Universidade Federal de Minas Gerais - Cedeplar/UFMG, Belo Horizonte-MG, Brasil (francis@cedeplar.ufmg.br).

** Departamento de Demografia da Universidade Federal de Minas Gerais - Cedeplar/UFMG, Belo Horizonte-MG, Brasil (moema@cedeplar.ufmg.br).
} 


\section{Introdução}

Há algumas décadas, a dinâmica da mortalidade tem mostrado que a esperança de vida de período, usada convencionalmente, não descreve a verdadeira experiência de vida da população presente em determinado ano (BONGAARTS; FEENEY, 2002; GUILLOT, 2003; SCHOEN; CANUDAS-ROMO, 2005; GOLDSTEIN; WACHTER, 2006). Para melhor compreender essa realidade, é preciso entender, formalmente, como a medida de longevidade e a experiência de vida populacional são analisadas.

Entre as várias medidas de mortalidade, a esperança de vida destaca-se por ser uma das mais utilizadas. Esse indicador sintetiza a experiência de vida de um grupo de indivíduos, geralmente nascidos no mesmo lugar e no mesmo período, que denominamos de coorte. De fácil interpretação, a esperança de vida expressa o número médio de anos a ser vivido pela coorte a partir de determinada idade (PRESTON; HEUVELINE; GUILLOT, 2001).

A estimativa do tempo médio de vida se dá por meio da tabela de sobrevivência, tão bem conhecida pelos demógrafos e atuários. A construção dessa tabela requer o acompanhamento de todos os membros de uma coorte, desde o nascimento até a morte, e exige também a estimativa das taxas de mortalidade em cada idade, calculadas à medida que os óbitos ocorrem. No entanto, aguardar a extinção de uma coorte tornaria esse estudo extremamente longo, chegando a ultrapassar um século de observação. Por isso, é utilizada a frequência de mortes observada em um único período como forma alternativa ao acompanhamento das coortes. Nesse caso, as taxas correntes verificadas no período fornecem uma aproximação da experiência de vida de uma coorte hipotética submetida ao padrão de mortalidade existente.

Contudo, quando há melhorias contínuas das condições de vida, a mortalidade sofre mudanças constantes no tempo. Portanto, as taxas observadas em um único período não retratam a esperança de vida real das pessoas vivas naquele período, pertencentes a diversas coortes que, provavelmente, estiveram expostas a diferentes regimes de mortalidade ao longo da vida. Nesse caso, se quiser entender como se desenvolve o processo de melhoria da longevidade em qualquer população, é necessário estudar o tempo médio de vida de suas coortes. Em face desses fatos, conclui-se que a esperança de vida de período não fornece informação da longevidade de qualquer coorte real, a não ser quando a mortalidade é constante no tempo.

A esperança de vida de uma coorte hipotética também não representa os prováveis ganhos de sobrevivência que tendem a ocorrer e a beneficiar a população futuramente. Logo, pode-se afirmar que a esperança de vida estimada por meio das taxas correntes de mortalidade se torna menor do que a esperança de vida real. Quando se compararam as estimativas de período, que são convencionalmente usadas, com as de coorte, conclui-se que viveremos mais do que pensamos.

A discussão acerca das limitações da esperança de vida é de suma importância para o desenvolvimento de diversas áreas de conhecimento. Essa medida-resumo da mortalidade é frequentemente empregada na análise das variações geográficas e temporais da longevidade 
das populações, assim como na comparação dos níveis de sobrevivência. O indicador de longevidade, relevante na avaliação dos níveis de saúde de uma população, também tem papel fundamental nos processos de planejamento, gestão e avaliação de políticas de saúde, de previdência social, entre outras, além de se relacionar à demografia dos negócios, como oferta de serviços, atualização de metas, cálculos atuariais, etc.

No presente trabalho, procura-se mostrar que, apesar de a tabela de sobrevivência de período não descrever nenhuma coorte em particular, a esperança de vida de período pode ser vista como um indicador defasado do tempo médio de vida de alguma coorte passada. Para tanto, é projetada a mortalidade para o Brasil, por sexo, visando determinar as esperanças de vida ao nascer de período e de coorte entre 1980 e 2050. Isso permitirá transladar empiricamente os resultados obtidos e analisar a evolução da correspondência entre ambas as medidas.

\section{Medidas de longevidade}

Discussões recentes sobre a dinâmica da mortalidade têm questionado o uso da esperança de vida de período como a medida de longevidade mais adequada para indicar as condições correntes de mortalidade. Surgiram então várias propostas para contornar esse problema que podem ser divididas em duas linhas. A primeira configura-se pelo estudo de medidas de longevidade alternativas que será discutida nesta seção e a segunda pela translação da medida convencional apresentada na seção seguinte.

Formalmente, a esperança de vida ao nascer de coorte pode ser calculada pelas taxas de mortalidade historicamente conhecidas ou projetadas. Porém, sua estimativa apresenta a desvantagem de exigir uma longa série de dados, de aproximadamente um século, e está sujeita a falhas nos pressupostos da projeção. Por definição, a esperança de vida ao nascer de coorte é dada por:

$$
e_{0}^{c}(t)=\int_{0}^{\infty} \exp \left\{-\int_{0}^{a} \mu(x, t+x) d x\right\} d a
$$

onde $\mu(x, t+x)$ é a força de mortalidade relativa à idade $x$, observada no tempo $t+x$.

Sob a perspectiva de coorte, uma vez que a idade e o tempo movem-se no mesmo sentido e sincronizados, a esperança de vida ao nascer fornece o resultado verossímil das melhorias nas condições de vida em uma população. Ou seja, o indicador de longevidade de coorte oferece informações reais sobre os níveis e padrões da mortalidade, bem como sobre suas variações temporais.

Já a esperança de vida ao nascer de período refere-se a uma medida hipotética, obtida pelas condições correntes de mortalidade prevalentes em um único período, sendo matematicamente definida como:

$$
e_{0}^{p}(t)=\int_{0}^{\infty} \exp \left\{-\int_{0}^{a} \mu(x, t) d x\right\} d a
$$


onde $\mu(x, t)$ é a força de mortalidade relativa à idade $x$, observada no tempo $t$.

Por outro lado, a esperança de vida ao nascer de período somente representaria a experiência de vida real de uma coorte recém-nascida caso todos os seus membros experimentassem o regime de mortalidade corrente, do seu nascimento até sua morte, inalteradamente (VAUPEL, 2002). Mas não é isso o que ocorre atualmente, visto que, quando a mortalidade declina, a estimativa de período torna-se menor do que a longevidade da coorte nascida no mesmo momento.

Logo, não se pode usar o indicador tradicional como um estimador apurado do tempo médio de vida da coorte recém-nascida. Tal resultado dá início a uma discussão de grande relevância demográfica: qual medida de longevidade é mais adequada quando a mortalidade está caindo?

Bongaarts e Feeney (2002) iniciam essa reflexão mostrando que, nas atuais circunstâncias, a esperança de vida de período sofre distorções no tempo e torna-se, portanto, insatisfatória a análise das condições correntes de mortalidade. A fim de corrigir as oscilações nas taxas específicas de mortalidade decorrentes do adiamento da morte ocorrido no período, os autores propõem um ajuste na esperança de vida convencional.

A esperança de vida ao nascer ajustada ao tempo pode ser descrita da seguinte forma:

$$
e_{0}^{\star}(t)=\int_{0}^{\infty} \exp \left\{-\int_{0}^{a} \frac{\mu(x, t)}{1-r(t)} d x\right\} d a
$$

sendo $r(t)$ a taxa de adiamento à morte segundo o modelo de mudanças proporcionais da mortalidade de Gompertz.

O novo indicador de longevidade de Bongaarts e Feeney (2002), denominado esperança de vida ajustada ao tempo, foi modelado para países desenvolvidos com elevada esperança de vida, onde se observam regularidades no processo de mudanças da mortalidade. Nesses países, quase a totalidade dos recém-nascidos sobrevive às idades adultas, além de serem percebidas melhorias similares da sobrevivência em todas as idades, a partir dos 30 anos. Nesse sentido, os pressupostos dos autores são basicamente os seguintes: mortalidade infanto-juvenil inexistente; melhorias da mortalidade proporcionais; população fechada à migração; e número de nascimentos constante sobre o tempo.

A esperança de vida de período ajustada ao tempo também é hipotética no tocante ao seu significado (BONGAARTS; FEENEY, 2006), pois nenhuma coorte em particular experimentará o conjunto de taxas ajustadas de mortalidade. A ideia principal dos autores é sintetizar o que ocorreria com o indicador de longevidade de período se, logo após o adiamento da morte, houvesse estabilização da mortalidade. Desse modo, a interpretação alternativa da esperança de vida ajustada ao tempo expressa qual seria a esperança de vida de período, caso não acontecesse mudança no tempo dos eventos, em uma população estacionária equivalente sob as condições correntes de mortalidade.

Outra medida de longevidade amplamente discutida é o tempo médio de vida transversal (Cross Sectional Average Length of Life - CAL - BROUARD, 1986; GUILLOT, 
2003). CAL refere-se a apenas um período em particular, mas leva em conta as condições correntes de mortalidade sob as quais as várias coortes presentes na população estão sujeitas. Essa medida pode ser descrita como:

$$
C A L(t)=\int_{0}^{\infty} l_{c}(a, t) d a
$$

onde $l_{c}(a, t)$ é a proporção de sobreviventes à idade $a$, no tempo $t$, de uma coorte nascida em $t-a$.

Esse novo indicador de longevidade baseia-se unicamente na estrutura etária populacional e pode ser compreendido como o tempo médio vivido no período, por aquelas coortes presentes na população.

Diferentemente da esperança de vida de período, que pode ser vista como a soma da proporção dos sobreviventes de uma coorte hipotética, CAL envolve a proporção de sobreviventes das várias coortes presentes na população nesse período em particular. Podese dizer, então, que CAL leva em conta as experiências reais vivenciadas por essas coortes (ou seja, as condições correntes reais de mortalidade), enquanto a esperança de vida de período sintetiza, exclusivamente, as taxas correntes.

CAL também denota a esperança de vida ao nascer de uma coorte, cujas vantagens e desvantagens relativas à sobrevivência das demais coortes presentes no período são iguais (GUILLOT, 2003). Assim, essa medida de longevidade pode ser considerada a experiência de vida de uma coorte média da população.

A construção de CAL requer informações históricas da mortalidade detalhadas por um período considerável de tempo, contrastando com a facilidade e simplicidade da esperança de vida tradicional. Em contrapartida, CAL não exige aguardar a extinção de todos os indivíduos da população para ser estimada da mesma forma que a esperança de vida geracional demanda.

Outra medida de longevidade também muito conhecida é a idade média à morte (Mean Age at Death - MAD), que se baseia na distribuição etária dos óbitos e pode ser entendida como o tempo médio vivido por aquelas pessoas que morreram em um dado período. Desse modo, MAD é definida por:

$$
M A D(t)=\frac{\int_{0}^{\infty} a d_{c}(a, t) d a}{\int_{0}^{\infty} d_{c}(a, t) d a}
$$

onde $d_{c}(a, t)$ é o número de óbitos ocorridos na idade $a$, no tempo $t$, de uma coorte nascida em $t-a$.

MAD se mostra uma das medidas mais simples de serem calculadas, porém, apresentase diretamente dependente da estrutura etária populacional e, portanto, mais suscetível às variações na sua distribuição.

Somando-se à discussão, Schoen e Canudas-Romo (2005) propõem outra medida de longevidade denominada esperança de vida de coorte média (Average Cohort Life Expectancy - ACLE). Esse estimador representa a média ponderada das esperanças de vida ao nascer 
das coortes presentes no período, em que os pesos são dados pelas probabilidades de sobrevivência da coorte até a idade observada no período. ACLE é representada por:

$$
A C L E(t)=\frac{\int_{0}^{\infty} l_{c}(a, t) e_{0}^{c}(t-a) d a}{\int_{0}^{\infty} l_{c}(a, t) d a}
$$

onde $e_{0}^{c}(t-a)$ é a esperança de vida da coorte nascida em $t-a$.

ACLE é um poderoso estimador do tempo médio de vida populacional (GOLDSTEIN; WACHTER, 2006), uma vez que é diretamente definida pela longevidade das coortes atuais. Porém, ACLE exige uma longa série histórica da mortalidade, de aproximadamente dois séculos. Além disso, para ser calculada no estado atual, essa medida requer projeções da dinâmica da mortalidade.

Embora as medidas de longevidade (esperança de vida de coorte, esperança de vida de período, esperança de vida ajustada ao tempo, CAL, MAD e ACLE) visem uma estimativa do tempo médio de vida, elas não são diretamente comparáveis, visto que cada uma se propõe a responder diferentes tipos de questões (WACHTER, 2005). Contudo, vale lembrar que, fixando a mortalidade a partir de determinado período ou de uma coorte específica, todas as medidas tenderiam ao mesmo nível, ainda que em ritmos distintos. Ademais, em uma situação de estabilidade, não haveria diferença no valor estimado por essas medidas de longevidade.

\section{Diferencial e defasagem}

As atuais condições de vida indicam um prolongado declínio da mortalidade, levando-nos a crer que os futuros níveis da mortalidade serão mais baixos do que os atuais. Em outras palavras, pode-se dizer que as taxas correntes de mortalidade não serão observadas pelos sobreviventes nos próximos anos, nem mesmo pelos recém-nascidos no próprio ano. Com isso, conclui-se que o tempo médio de vida corrente, calculado por meio da esperança de vida ao nascer de período, subestimará a longevidade da coorte recém-nascida.

Enquanto a população passar pelo processo de melhorias da mortalidade, a curva da esperança de vida ao nascer de período, em função do tempo, estará sempre abaixo da curva da esperança de vida ao nascer de coorte, como mostrado na Figura 1.

FIGURA 1

Ilustração teórica do diferencial e da defasagem entre a esperança de vida ao nascer de período e de coorte, quando há declínio da mortalidade

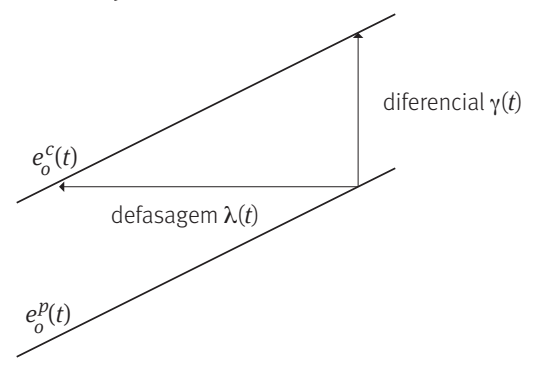

Fonte: Goldstein e Wachter (2006). 
A distância vertical entre as duas curvas fornece o diferencial $y(t)$ entre as duas medidas de longevidade observadas no ano corrente. Formalmente, $y(t)$ informa quantos anos a esperança de vida do período observado difere da esperança de vida da coorte nascida no mesmo período. É possível afirmar também que o diferencial fornece o bônus que a coorte recém-nascida recebe da futura melhoria na mortalidade (GOLDSTEIN; WACHTER, 2006). Empiricamente, encontra-se o diferencial da seguinte maneira:

$$
\gamma(t)=e_{0}^{c}(t)-e_{0}^{p}(t)
$$

Já a distância horizontal entre as curvas das esperanças de vida ao nascer fornece a defasagem $\lambda(t)$ entre o ano corrente, no qual se obtém a estimativa de período, e o ano de nascimento da coorte, cuja estimativa do tempo médio de vida é equivalente. Desse modo, $\lambda(t)$ mostra quanto tempo é preciso regredir para se encontrar uma coorte com a mesma esperança de vida observada no período atual. Pode-se dizer que a defasagem empírica é representada por:

$$
\lambda(t)=\min \left\{\lambda \mid e_{0}^{c}(t-\lambda)=e_{0}^{p}(t)\right\}
$$

Em vista dessa correspondência, observa-se que a esperança de vida ao nascer de período pode ser considerada um indicador defasado da longevidade de coorte. Verifica-se também que a translação da esperança de vida de período está diretamente relacionada com a defasagem, uma vez que encontrar a coorte equivalente implica localizar no tempo o ano de correspondência da estimativa de período. Empiricamente, esse processo exige o conhecimento de longas séries estatísticas de mortalidade, incluindo a experiência de vida passada e projetada.

\section{Série estatística de mortalidade}

Para mostrar que a medida convencional de longevidade pode ser vista como um indicador defasado do tempo médio de vida de alguma coorte passada, foram utilizadas as estimativas e projeções das tabelas de sobrevivência oficiais do Brasil, divulgadas pelo Instituto Brasileiro de Geografia e Estatística - IBGE. Essa série de tábuas é resultante de uma ampla discussão durante uma oficina de trabalho entre técnicos da Coordenação de População e Indicadores Sociais (Copis/DPE/IBGE) e do Centro Latinoamericano y Caribeño de Demografía (Celade/Cepal/Nações Unidas), realizada entre 24 e 28 de março de 2003, em Santiago, Chile. Os dados históricos básicos utilizados na sua construção foram obtidos a partir das Estatísticas Vitais e dos Censos Demográficos brasileiros. As tábuas disponibilizadas pelo IBGE são construídas por sexo e cobrem o período de 1980 a 2050, anualmente.

No entanto, a série histórica brasileira do IBGE não é suficiente para o cálculo das medidas de longevidade de coorte no período em que se pretende analisá-las (1980-2050), pois, para a construção da tabela de sobrevivência de coorte, é preciso o conjunto das taxas de mortalidade observadas sobre um longo período de tempo.

Assim, foram projetadas as taxas específicas de mortalidade para o período de 2051 a 2130, segundo a metodologia da tabela limite. ${ }^{1}$ Para isso, levantou-se a suposição de que a

1 Para mais detalhes, ver Pujol (1984). 
mortalidade tenderá a cair indeterminadamente, porém em ritmo cada vez mais lento. Essa queda se dará principalmente nas idades mais velhas e implicará aumento da esperança de vida mais acentuado no início do processo.

A estrutura de mortalidade projetada, para esse período, obtém-se por meio dos logitos das taxas específicas de mortalidade $m(x, t)$ da tábua de mortalidade de 2050 e da tábua de mortalidade limite de 2100 . Essa projeção pode ser descrita da seguinte forma:

$$
y_{x}^{K}=\frac{t_{L}-t_{K}}{t_{L}-t_{I}} y_{x}^{I}+\frac{t_{K}-t_{I}}{t_{L}-t_{I}} y_{x}^{T}
$$

tal que:

$$
y_{x}=\frac{1}{2} \ln \frac{1-m(x, t)}{m(x, t)}
$$

onde $y_{x}^{K}$ é o logito de $m(x, t)$ projetada para a tábua de mortalidade para o ano $t_{k} ; y_{x}^{I}$ e $y_{x}^{T}$ são os logitos de $m(x, t)$ da tábua de mortalidade inicial, do ano $t_{I}$ (que neste caso é 2050) e da tábua limite, do ano $t_{L}$ (que neste caso é 2100), respectivamente.

As estimativas usadas para 2050 foram projetadas pelo IBGE e as de 2100 construídas com base nas informações das tabelas de sobrevivência limites propostas pelo U. S. Bureau of the Census. Com base nas taxas específicas de mortalidade estimadas e projetadas para 1980 a 2130, foram produzidas tabelas de sobrevivência para as coortes brasileiras, cujo ano de nascimento varia entre 1980 e 2050, por sexo. Consequentemente, por meio dessas tábuas, estimou-se a esperança de vida ao nascer de coorte para o Brasil nas mesmas condições.

\section{Tabela de sobrevivência de coorte}

As tabelas de sobrevivência construídas para coorte ou geração real seguem, basicamente, os mesmos passos daquela construída para período, em que são estimadas várias funções de mortalidade por idade que descrevem o comportamento vital de um grupo de indivíduos (PITACCO et al., 2009; PRESTON; HEUVELINE; GUILLOT, 2001). A grande diferença entre ambas está no conjunto de taxas específicas de mortalidade usado nos diversos cálculos das tabelas. Para as estimativas de coorte, a tabela é gerada com base no conjunto de taxas de mortalidade vivenciadas por um grupo real de indivíduos, ao passo que a tabela de período é criada pelo conjunto de taxas observadas em apenas um período (geralmente, um ano).

As taxas de mortalidade referem-se ao risco de morte em cada idade ou em cada grupo etário e correspondem ao quociente entre o total de óbitos ocorridos em determinado ano, em cada idade ou grupo etário, e a população correspondente no meio do ano. Por meio dessas taxas, construíram-se as probabilidades de morte e as probabilidades de sobrevivência. Assim:

$q(x, t+x)=1-\exp (-m(x, t+x))$ 
representa a probabilidade de um indivíduo de idade exata $x$ vir a morrer antes de completar $x+1$ anos, ${ }^{2}$ dado que $m(x, t+x)$ expressa a taxa específica de mortalidade na idade $x$, observada no tempo $t+x$. Note-se que, como estamos tratando de coorte, as funções de mortalidade sempre referem-se ao momento real em que os indivíduos nascidos em $t$ se expõem a risco de morte (em $t+x)$.

Para encontrar o número de sobreviventes a determinada idade $l(x, t+x)$, basta desenvolver a expressão (10) a partir de $x=O$ (ou seja, do número de nascimentos em $t$ ) até alcançar a idade desejada:

$$
l(x, t+x)=l(x-1, t+x-1)(1-q(x-1, t+x-1))
$$

O número de óbitos $d(x, t+x)$ ocorridos entre os indivíduos de uma coorte resulta da diferença entre os sobreviventes observados em dois anos consecutivos ou entre duas idades seguidas, já que na perspectiva de coorte as variáveis tempo e idade progridem no mesmo sentido e sincronizadas. Dessa forma:

$$
d(x, t+x)=l(x+1, t+x)-l(x, t+x)
$$

A principal medida estimada pela tábua de mortalidade e também objeto do presente estudo é a esperança de vida ${ }^{3}$ ao nascer, que em termos discretos é dada por:

$$
e_{0}^{c}(t)=\frac{\sum_{x=0}^{\omega} L(x, t+x)}{l(0, t)}
$$

onde $L(x, t+x)$ é o tempo a ser vivido pelos sobreviventes da coorte, nascida em $t$, entre as idades $x$ e $x+1$. $L(x, t+x)$, por sua vez, mantém a seguinte relação:

$$
L(x, t+x)=\frac{d(x, x+t)}{m(x, x+t)}
$$

Observe-se que o cálculo da esperança de vida ao nascer exige o acompanhamento da coorte em estudo de seu nascimento até sua extinção, tal como requer a estimativa das taxas específicas de mortalidade ao longo dos anos. Por isso, como afirmado ao início deste trabalho, a construção da tábua de mortalidade de coorte torna o estudo da longevidade extremamente demorado.

As tabelas de sobrevivência de período podem fornecer um ponto de partida na análise da longevidade quando as tabelas de coorte não são factíveis. No entanto, quando a mortalidade declina, as tabelas de sobrevivência de período revelam níveis de mortalidade mais elevados do que aqueles expressos pelas tabelas de coorte. Por isso, o uso das estimativas correntes deveria ser restrito a estudos prospectivos de curto e médio prazos (no máximo dez anos) (PITACCO et al., 2009). Alguns estudos demográficos prospectivos de longo prazo (tais como planejamentos atuariais, previdenciários e de seguridade, que geralmente exigem o

\footnotetext{
2 A probabilidade de sobrevivência entre as idades exatas $x$ e $x+1$ é $p(x, t+x)=1-q(x, t+x)$.

3 Analogamente, pode-se construir a esperança de vida a partir de qualquer idade; basta iniciar o somatório em (12) com a idade de interesse.
} 
conhecimento antecipado das tendências da mortalidade) deveriam ser realizados com base nas tabelas de sobrevivência de coorte.

\section{Resultados}

Para a análise das tendências da mortalidade no Brasil, confiou-se num cenário em que as esperanças de vida crescem acentuadamente nas primeiras décadas e, com o passar do tempo, os ganhos da sobrevivência tendem a se reduzir (Gráfico 1). A diminuição no ritmo das melhorias na mortalidade é natural à medida que a longevidade se estende. Quando os níveis de mortalidade são elevados, principalmente a mortalidade infantil, há muito para se melhorar. Porém, quando os níveis se encontram baixos, perceber algum ganho na sobrevivência se torna difícil.

Não foram projetados impactos ou choques externos na mortalidade, para o período 1980-2050, implicando, portanto, que ambas as curvas das esperanças de vida sejam suaves. Essas curvas possuem ainda concavidade voltada para baixo e apresentam crescimento constante no tempo. Assim, entende-se que o processo de ganhos da sobrevivência será contínuo, não havendo estabilização das estimativas do tempo médio de vida nesse período.

Observa-se, no Gráfico 1, que as curvas das esperanças de vida, tanto de período quanto de coorte, não são paralelas entre si. Isto significa que o ritmo de crescimento de cada estimativa é diferente e o processo de queda na mortalidade torna-se complexo de ser descrito. A medida de longevidade de coorte é mais sensível ao declínio da mortalidade no início da queda (período não apresentado neste trabalho), já que houve um distanciamento entre as curvas, enquanto a medida de período é mais sensível às mudanças da mortalidade quando o ritmo da queda desacelera (como descrito no Gráfico 1), o que explica a distância decrescente entre as curvas.

\section{GRÁFICO 1 \\ Tendência das esperanças de vida ao nascer de coorte (EV coorte) e de período (EV período) e defasagem e diferenciais resultantes estimados, por sexo \\ Brasil - 1980-2050}

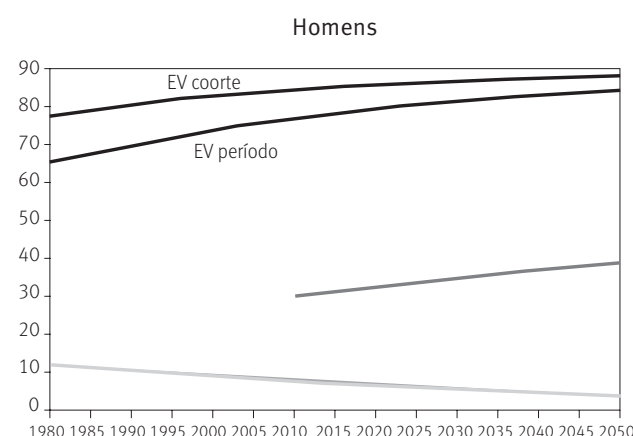

— Defasagem empírica

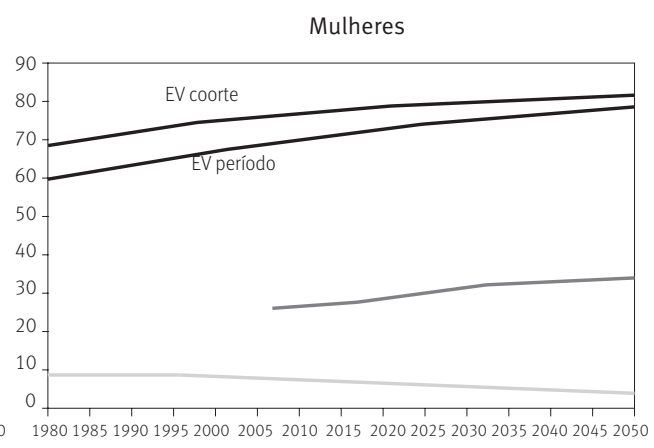

Diferencial empírico 
Sabendo que as taxas de mortalidade observadas no período produzem um valor cada vez mais próximo da esperança de vida de coorte, gerada por meio do conjunto de taxas experimentadas ao longo dos anos, o diferencial torna-se decrescente com o tempo. O declínio acentuado da curva do diferencial somente confirma o fato de que as esperanças de vida se aproximam com o passar dos anos. Segundo nossa projeção, este resultado leva a crer que a medida convencional de período se mostrará um indicador acurado da esperança de vida de coorte em poucas décadas. No entanto, a defasagem indica justamente o oposto.

No Gráfico 1, verifica-se que as curvas da defasagem crescem com o tempo e mostram uma tendência linear com pequena inclinação. Em outras palavras, pode-se dizer que, enquanto as melhorias na mortalidade persistirem, é necessário regredir cada vez mais para encontrar uma coorte cuja longevidade seja equivalente à estimativa de período. Logo, ao contrário do que se pode pensar, mesmo que a esperança de vida de período tenda ao mesmo nível da esperança de vida de coorte, não é possível afirmar que a medida convencional de período seja um bom indicador da longevidade da coorte recém-nascida, uma vez que a defasagem mostra um distanciamento progressivo da coorte equivalente.

As coortes equivalentes experimentam uma mistura de altas e baixas taxas de mortalidade, comparativamente àquelas observadas no período de correspondência. Os altos níveis de mortalidade correspondem às taxas passadas e os baixos níveis referem-se às taxas futuras. Quando o ritmo de melhoria da mortalidade cai, as taxas vivenciadas pelas coortes no passado continuam elevadas em relação aos níveis correntes, enquanto as taxas futuras não se tornam tão expressivamente mais baixas. Assim, mesmo que o ganho na evolução da esperança de vida de período seja pequeno, as estimativas de coorte perceberão ganhos menores ainda, o que leva a um distanciamento da correspondência entre período e coorte.

A defasagem também pode ser analisada em outro sentido, ou seja, o tempo necessário para a esperança de vida de período alcançar o nível da esperança de vida da coorte recém-nascida. Quanto maior for o ganho da sobrevivência em determinado período, menos tempo será preciso para que a medida de período se torne equivalente à esperança de vida da coorte em questão. Em contrapartida, quanto menor for o ganho observado no período, mais tempo será necessário para encontrar a correspondência. Logo, os resultados não são contraditórios. Com menores ganhos, as esperanças de vida de período e coorte do mesmo ano se aproximam, mas a equivalência torna-se mais difícil.

É importante destacar que não há estimativas da defasagem para todo o intervalo em estudo. A correspondência entre as esperanças de vida de período e de coorte não pode ser encontrada para os primeiros anos, uma vez que a série estatística aqui utilizada conta com dados de mortalidade unicamente entre 1980 e 2050. Dada a inexistência de informações sobre as taxas específicas de mortalidade antes de 1980, não há meios de se calcular a esperança de vida de coorte para anos anteriores a 1980, que, por sua vez, forneceria o tempo de defasagem.

A série da defasagem estende-se por intervalos diferentes para cada sexo. Para os homens, foi possível estabelecer as estimativas da defasagem a partir do ano (período) de 
2007 e, para as mulheres, somente a partir de 2010. A baixa esperança de vida masculina, comparativamente à feminina, tornou possível a correspondência da esperança de vida de período masculina para um maior número de anos. Nesse sentido, as coortes equivalentes masculinas são mais próximas dos períodos de referência do que as femininas. A acentuada diferença nos níveis de longevidade entre os sexos é decorrente dos altos níveis de mortalidade masculina, principalmente por causas externas. A despeito disso, os resultados mostram que as tendências de crescimento das esperanças de vida ao nascer de período e de coorte são percebidas semelhantemente entre homens e mulheres.

Na Tabela 1 apresenta-se a translação da esperança de vida ao nascer de período para o Brasil, por sexo, determinando qual coorte de nascimento teria sua longevidade equivalente à estimativa de período. Por exemplo, a esperança de vida ao nascer calculada para as mulheres em 2010 é equivalente à esperança de vida de uma coorte feminina nascida em 1980. Assim, a esperança de vida estimada para a coorte hipotética recém-nascida em 2010 estaria, na realidade, estimando a longevidade de uma coorte passada. Em outras palavras, pode-se dizer que as mulheres que alcançassem 30 anos em 2010 teriam sua esperança de vida ao nascer equivalente à estimativa do ano corrente, ao passo que aquelas com idade abaixo e acima de 30 anos teriam sua longevidade subestimada e sobreestimada, respectivamente.

TABELA 1

Translação da esperança de vida ao nascer de período, por sexo

Brasil - 2007-2050

\begin{tabular}{|c|c|c|c|c|c|}
\hline \multirow{2}{*}{ Período } & \multicolumn{2}{|c|}{ Coorte equivalente } & \multirow{2}{*}{ Período } & \multicolumn{2}{|c|}{ Coorte equivalente } \\
\hline & Homens & Mulheres & & Homens & Mulheres \\
\hline 2007,5 & 1981,2 & - & 2029,5 & 1998,0 & 1995,3 \\
\hline 2008,5 & 1982,1 & - & 2030,5 & 1998,8 & 1996,1 \\
\hline 2009,5 & 1982,9 & - & 2031,5 & 1999,4 & 1996,8 \\
\hline 2010,5 & 1983,8 & 1980,8 & 2032,5 & 2000,2 & 1997,4 \\
\hline 2011,5 & 1984,5 & 1981,6 & 2033,5 & 2001,0 & 1998,1 \\
\hline 2012,5 & 1985,3 & 1982,3 & 2034,5 & 2002,0 & 1998,8 \\
\hline 2013,5 & 1986,1 & 1983,0 & 2035,5 & 2003,0 & 1999,6 \\
\hline 2014,5 & 1987,0 & 1983,8 & 2036,5 & 2003,9 & 2000,2 \\
\hline 2015,5 & 1987,9 & 1984,6 & 2037,5 & 2004,8 & 2001,0 \\
\hline 2016,5 & 1988,7 & 1985,4 & 2038,5 & 2005,7 & 2001,9 \\
\hline 2017,5 & 1989,5 & 1986,2 & 2039,5 & 2006,6 & 2002,8 \\
\hline 2018,5 & 1990,3 & 1987,0 & 2040,5 & 2007,6 & 2003,6 \\
\hline 2019,5 & 1991,0 & 1987,9 & 2041,5 & 2008,4 & 2004,4 \\
\hline 2020,5 & 1991,8 & 1988,8 & 2042,5 & 2009,3 & 2005,2 \\
\hline 2021,5 & 1992,4 & 1989,5 & 2043,5 & 2010,1 & 2006,0 \\
\hline 2022,5 & 1993,0 & 1990,2 & 2044,5 & 2011,0 & 2006,9 \\
\hline 2023,5 & 1993,7 & 1990,9 & 2045,5 & 2012,0 & 2007,8 \\
\hline 2024,5 & 1994,4 & 1991,7 & 2046,5 & 2012,8 & 2008,5 \\
\hline 2025,5 & 1995,1 & 1992,5 & 2047,5 & 2013,6 & 2009,3 \\
\hline 2026,5 & 1995,9 & 1993,2 & 2048,5 & 2014,5 & 2010,1 \\
\hline 2027,5 & 1996,6 & 1993,9 & 2049,5 & 2015,3 & 2010,9 \\
\hline 2028,5 & 1997,3 & 1994,6 & 2050,5 & 2016,3 & 2011,8 \\
\hline
\end{tabular}

Fonte: IBGE (2005 e 2009a). 
As estimativas de longevidade construídas para período até meados da década de 2030 equivalem às estimativas de coortes nascidas no século passado. Já para as demais estimativas produzidas até 2050, a correspondência não ultrapassa a coorte de 2016. Esses resultados relacionam-se diretamente com a defasagem. No início da translação, a defasagem é de 26,3 anos para os homens e de 29,7 anos para as mulheres, enquanto, ao final do intervalo em estudo, o distanciamento se amplia, passando para 34,2 e 38,7 anos para homens e mulheres, respectivamente. Enfim, com o passar dos anos, as coortes equivalentes tornam-se cada vez mais remotas.

\section{Discussão}

Em uma população cuja mortalidade esteja fixa, a experiência de vida geracional sempre se repete, tornando possível observar em um único período todas as funções de mortalidade que geram a esperança de vida de qualquer coorte. Assim, não haveria diferença entre as estimativas obtidas na análise longitudinal e aquelas alcançadas na análise transversal da população. Entretanto, com o processo de mudanças da sobrevivência, observa-se que as taxas específicas de mortalidade se alteram constantemente, fazendo com que não seja possivel a correspondência entre as condições correntes de mortalidade e as verdadeiras experiências de vida das coortes.

Aprofundando a reflexão sobre as mudanças da sobrevivência, constatou-se que nenhuma coorte presente em determinado ano absorverá todas as melhorias percebidas nesse período, pois o único ganho real alcançado pelas pessoas será aquele observado para a sua idade. Caso as condições de mortalidade continuem melhorando, os indivíduos que sobreviverem aos períodos seguintes continuarão se beneficiando de ganhos na longevidade. Nesse contexto, podem ser notadas duas situações: as coortes mais velhas, presentes na população, vivenciaram riscos de morte mais elevados do que as mais jovens, enquanto as coortes futuras vivenciarão riscos mais baixos do que as atuais.

Mesmo a mortalidade se estabilizando, a esperança de vida de período somente estimará o tempo médio de vida da coorte recém-nascida, uma vez que as demais coortes experimentaram níveis de mortalidade diferentes nos anos anteriores. Além disso, até que todos os indivíduos nascidos previamente a essa estabilização morram, a longevidade calculada pelas taxas correntes de mortalidade não será uma estimativa acurada para todas as coortes sobreviventes no período.

Caso as condições de mortalidade se fixassem a partir de algum momento, todas as coortes ali presentes perceberiam os mesmos ganhos relativos da sua idade atual em diante, embora nem todos os indivíduos fossem beneficiados por ganhos nas idades mais jovens. Portanto, quando a mortalidade sofre ou sofreu recentemente oscilações, a esperança de vida de período fornece indicadores enviesados sobre a longevidade das coortes reais.

Ao se considerar a natureza da mortalidade de países contemporâneos, as recentes metodologias exigem suposições, muitas vezes, irrealistas, pois, dependendo do estágio 
de desenvolvimento em que cada país se encontra, não é uma tarefa fácil e generalizável descrever o processo de mudanças da mortalidade em vista do atual padrão de sobrevivência e de possíveis melhorias das condições de vida.

A existência do efeito tempo na esperança de vida de período também é questionável, uma vez que esse conceito é originário do campo da fecundidade, em que se pode perceber claramente a diferença entre o efeito tempo e o efeito quantum. Na fecundidade, a proposta original de ajuste se dá na taxa de fecundidade total (medida de quantum), diferentemente da proposta da mortalidade, que seria na esperança de vida de período (medida de tempo). Já que a mortalidade é uma componente demográfica puramente de tempo, a adaptação da ideia de distorção para a análise da mortalidade não é amplamente aceita.

Para Rodriguez (2006), mesmo que reduções das taxas de mortalidade correspondam ao retardamento dos óbitos, o quantum no nível individual é sempre um, pois cada pessoa morre apenas uma vez. Não há risco de interpretações incorretas. Uma redução nas taxas de mortalidade somente pode significar que as pessoas estão adiando a idade média à morte e que, portanto, não há necessidade de ajustes na esperança de vida de período.

Os novos estimadores de longevidade, em geral, visam analisar as condições correntes de mortalidade, mas esse termo também é alvo de grande discussão. Segundo Vaupel (2002), as condições correntes de mortalidade referem-se ao regime das condições de saúde prevalentes na população, que, por sua vez, são complexas e difíceis de serem mensuradas. Para o autor, devem-se levar em conta quaisquer fatores que influenciem o risco de morte, tais como nível educacional, exposição a doenças, dieta, tabagismo, genética, etc. Por outro lado, Bongaarts e Feeney (2008) entendem condições correntes de mortalidade como o regime de mortalidade a ser experimentado pela população estacionária equivalente, após a estabilização da mortalidade. Já para grande parte dos demógrafos, as condições correntes de mortalidade, no sentido mais popular da expressão, são expressas pelas taxas correntes de mortalidade.

Enfim, o termo condições correntes é definido segundo o objeto de estudo de cada pesquisador. Portanto, generalizando, conceituou-se neste trabalho a análise das condições correntes de mortalidade como toda medida que se propõe ao estudo transversal da longevidade.

Se pensarmos nos novos indicadores de longevidade por outra perspectiva, estaremos construindo uma medida alternativa à esperança de vida de período, a qual, por sua vez, também é uma medida alternativa de esperança de vida de coorte. Assim, estabelecer a relação entre a esperança de vida de período e de coorte é tão ou mais importante do que o estudo de novas medidas de longevidade. Porém, se desejamos estudar as condições correntes de mortalidade, em vez das experiências de vida das coortes, então, a alternativa mais razoável continua sendo empregar a esperança de vida de período, por ser facilmente calculada e amplamente difundida.

Em se tratando da medida geracional, vale lembrar que a projeção do declínio da mortalidade, estabelecida por uma tabela limite, pode subestimar ou sobre-estimar as medidas 
reais de longevidade em longo prazo. Mesmo baseando-se nas tendências observadas na população brasileira, a incerteza sobre o ritmo de melhorias a ser experimentado nas próximas décadas está presente em qualquer projeção que se faça. Entretanto, essa limitação não leva a conclusões absurdas; pelo contrário, os resultados alcançados conduzem a um ponto de partida factível. Ao que tudo indica, caminha-se no sentido da consolidação do processo de envelhecimento populacional e da retangularização da curva de sobrevivência, perfil típico de países desenvolvidos.

\section{Conclusão}

Não é surpresa alguma encontrar nas estimativas oficiais a evidenciada melhoria da esperança de vida ao nascer. Isso porque a esperança de vida, principalmente ao nascer, é indicadora do nível de desenvolvimento do país, refletindo todo o conjunto de condições favoráveis ou desfavoráveis ali presentes. O registro do declínio histórico da mortalidade no Brasil, pelas instituições governamentais brasileiras, sempre se deu sob a perspectiva de período. Portanto, a análise das tendências da longevidade do país limita-se às informações oficiais construídas para período. Contudo, enquanto o Brasil experimentar melhorias nas condições de vida, a tabela de sobrevivência de período não descreverá nenhuma coorte real.

Nesse contexto, os estudiosos da área apresentaram propostas alternativas à estimativa do tempo médio de vida, cada qual se propondo a responder diferentes tipos de questões. As recentes propostas trazem alguns pressupostos incompativeis com o atual contexto brasileiro, pois, na maioria das vezes, são delineados para países desenvolvidos cuja estrutura de mortalidade mostra-se diferente da nossa. Além disso, as novas medidas de longevidade exigem longas séries históricas sobre a dinâmica populacional, da qual ainda não existe um registro tão completo.

Os novos estimadores de longevidade têm como ideia principal analisar as condições correntes de mortalidade, ao passo que o objetivo aqui é abordar a experiência de vida real das coortes presentes na população. A proposta não visa discutir como construir o tempo médio de vida, mas sim o que deve ser utilizado na sua estimativa. A síntese da esperança de vida por meio da construção da tabela de sobrevivência mostra-se tão vantajosa como sempre foi, porém, o emprego das taxas específicas de mortalidade de período não é o único meio de estimar a longevidade populacional. Ao que tudo indica, a esperança de vida convencional não cairá em desuso, dada a facilidade de seu cálculo e a sua ampla difusão. Por isso, mostrou-se que ela pode ser vista como um indicador defasado do tempo médio de vida de alguma coorte passada.

A translação da esperança de vida ao nascer de período, na perspectiva de coorte, é um recurso proveitoso para fornecer indicadores de quanto se configura o bônus relativo às futuras melhorias na mortalidade. Já a medida de coorte oferece estimativas reais, ou pelo menos mais próximas, da longevidade de um grupo específico de indivíduos. Ao serem projetadas as tabelas de sobrevivência de coorte, obtêm-se, além da esperança de vida ao 
nascer, a sobrevida em qualquer idade e muitas outras funções de mortalidade para um grupo real de pessoas.

Apesar de a mortalidade se encontrar instável e de ser difícil afirmar sobre sua possível estabilidade ou quase-estabilidade, vale lembrar que essa componente demográfica ganha papel cada vez mais importante sobre o processo de envelhecimento e de crescimento populacional. Por isso, a discussão sobre medidas de longevidade, tanto transversal quanto longitudinal, tem se intensificado.

\section{Referências}

BONGAARTS, J. Five period measures of longevity. Demographic Research, v. 13, p. 547-558, 2005. BONGAARTS, J.; FEENEY, G. How long do we live? Population and Development Review, v. 28, n. 1 , p. 13-29, 2002.

Estimating mean lifetime. Proceedings of the National Academy of Sciences of the United States of America, v. 100, n. 23, p. 13127-13133, 2003.

The quantum and tempo life-cycle events. Vienna Yearbook of Population Research, p.115-151, 2006.

Afterthoughts on the mortality tempo effect. In: BARBI, E.; BONGAARTS, J.; VAUPEL, J. W. (Eds.). How long do we live? Demographic models and reflections on tempo effects. Hardcover: Springer, 2008, p. 263-269.

BROUARD, N. Structure et dynamique des populations. La pyramide de années vivre, aspects nationaux et exemples régionaux. Espaces, Populations, Socits, p.157-168, 1986.

CANUDAS-ROMO, V.; SCHOEN, R. Age-specific contributions to changes in the period and cohort life expectancy. Demographic Research, v. 13, p. 63-82, 2005.

FEENEY, G. Increments to life and mortality tempo. Demographic Research, v. 14, p. 27-46, 2006.

GOLDSTEIN, J. Found in translation? A cohort perspective on tempo-adjusted life expectancy. Demographic Research, v. 14, p. 71-84, 2006.

GOLDSTEIN, J. R.; WACHTER, K. Relationships between period and cohort life expectancy: gaps and lags. Population Studies, v. 60, n. 3, p. 257-269, 2006.

GUILLOT, M. The croos-sectional average length of life (CAL): a cross-sectional mortality misure that reflects the experience of cohorts. Population Studies, v. 57, n. 1, p. 41-54, 2003.

Tempo effects in mortality: an appraisal. Demographic Research, v. 14, p. 1-26, 2006.

HORIUCH, S. Tempo effect on age-specific death rates. Demographic Research, v. 13, p. 189-203, 2005.

IBGE - Instituto Brasileiro de Geografia e Estatística. Arquivo de entrada: PRE2003C. Rio de Janeiro, 2005.

Metodologia para construção da Tábua Completa de Mortalidade para a população do Brasil. Rio de Janeiro, 2009a. Disponível em: 〈http://ibge.gov.br/servidor_arquivos_est/〉. Acesso em: 5 fev. 2010.

. Notas técnicas: dinâmica demográfica e a mortalidade no Brasil no período 1998-2008. Rio de Janeiro, 2009b. Disponível em: «http://ibge.gov.br/home/estatistica/populacao/tabuadevida/2008/ default.shtm>. Acesso em: 5 fev. 2010. 
JUDSON, D. H.; POPOFF, C. L. Selected General Methods. In: SIEGEL, J. S.; SWANSON, D. A. (Eds.). The methods and materials of demography. San Diego: Elsevier Academic Press, 2004, p. 677-732.

LE BRAS, H. Mortality tempo versus removal of causes of mortality: opposite views leading to different estimations of life expectancy. Demographic Research, v. 13, p. 615-640, 2005.

LUY, M. Mortality tempo-adjustment: theorical considerations and an empirical application. Demographic Research, v. 15, p. 561-590, 2006.

OLIVEIRA, J. C.; ALBUQUERQUE, F. R. P. C. A evolução da esperança de vida no Brasil na última década do século XX: os ganhos e os diferenciais por sexo. Rio de Janeiro: Diretoria de Pesquisas DPE, Departamento de População e Indicadores Sociais - Depis/ IBGE, 2009. Disponível em: «http:// ibge.gov.br/servidor_arquivos_est/>. Acesso em: 5 fev. 2010.

PITACCO, E. et al. Modellig longevity dynamics for pensios and annuity business. Oxford: Oxford University Press, 2009. Cap. 2. Life tables, p. 45-88.

PRESTON, S. H.; HEUVELINE, P.; GUILLOT, M. Demography: measuring and modeling population processes. Oxford: Blackwell Publishers, 2001.

PUJOL, J. M. Procedimientos de proyección de la mortalidad utilizados en CELADE. In: CHACKIEL, J.; MACCIÓ, G. (Orgs.). Métodos para proyecciones demográficas. San José: Centro Latinoamericano y Caribeño de Demografía, 1984, p. 87-119.

RODRIGUEZ, G. Demographic translation and tempo effects: an accelerated failure time perspective. Demographic Research, v. 14, p. 85-110, 2006.

SCHOEN, R. Dynamic population models. Dordrecht: Springer, 2006. Cap.5. Longevity and dynamic mortality, p. 79-98.

SCHOEN, R.; CANUDAS-ROMO, V. Changing mortality and average cohort life expectancy. Demographic Research, v. 13, p. 117-142, 2005.

VAUPEL, J. W. Life expectancy at current rates vs. current conditions: a reflexion stimulated by Bongaarts an Feeney’s “How long do we live?” Demographic Research, v. 7, p. 365-378, 2002.

Lifesaving, lifetimes and lifetables. Demographic Research, v. 13, p. 597-614, 2005.

Turbulence in lifetables: demonstration by four simple examples. In: BARBI, E.; BONGAARTS, J.; VAUPEL, J. W. (Eds.). How long do we live? Demographic models and reflections on tempo effects. Hardcover: Springer, 2008, p. 270-279.

VAUPEL, J. W.; CANUDAS-ROMO, V. Decomposing change in life expectancy: a bouquet of formulas in honor of Nathan Keyfitz's 90th birthday. Demography, v. 40, n. 2, p. 201-216, 2003.

WACHTER, K. Tempo and its tribulations. Demographic Research, v. 13, p.201-222, 2005.

\section{Autoras}

Francismara Fernandes Guerra é doutoranda em Demografia no Departamento de Demografia da Universidade Federal de Minas Gerais - Cedeplar/UFMG e mestre em Demografia (francis@cedeplar. ufmg.br).

Moema Bueno Gonçalves Fígoli é doutora em Demografia e professora associada do Departamento de Demografia da Universidade Federal de Minas Gerais - Cedeplar/UFMG (moema@cedeplar.ufmg.br). 


\section{Abstract \\ Life expectancy and its relationship with indicators of longevity: a demographic study for Brazil, 1980-2050}

When mortality is constantly changing, the period life table fails to portray the real life experience of the present cohorts of the population. Therefore, the use of current mortality rates to estimate average lifetimes distorts the original concept of life expectancy. Longevity as a cohort measure should be estimated for a real group of people, but it usually applies to a hypothetical cohort. Given past and likely future reductions in death rates, the cohort life expectancy becomes higher than period life expectancy. Nevertheless, period life expectancy at birth can be viewed as a lagged indicator of the average lifetime of some past cohort (equivalent cohort). This paper explores the time, or lag, required to find this past cohort and the differences, or gaps, between life expectancies in a given year to determine the translation of the period measure from a cohort perspective. With a shrinking trend of gains in longevity, our results indicate that for both men and women in Brazil the gap shrinks with time, whereas the lag increases. That is, over the years, the current estimates of period and cohort tend to approximate one another whereas equivalent cohorts grow farther apart.

Keywords: Measures of longevity. Translation of life expectancy. Gap. Lag.

\section{Resumen}

\section{Esperanza de vida y su relación con indicadores de longevidad: un estudio demográfico para} Brasil, 1980-2050

Cuando la mortalidad se encuentra en constante cambio, la tabla de supervivencia de periodo no describe la verdadera experiencia de vida de las cohortes presentes en la población. En este sentido, el empleo de las tasas corrientes de mortalidad en la estimación del tiempo promedio de vida distorsiona el concepto original de esperanza de vida. Como una medida de cohorte, la longevidad debería estimarse para un grupo real de personas, pero usualmente se refiere a una cohorte hipotética. Dadas las reducciones pasadas de la mortalidad y las probables reducciones futuras, la esperanza de vida de cohorte se hace mayor que la de periodo. A pesar de ello, se puede considerar a esta última como un indicador desfasado del tempo medio de vida de alguna cohorte pasada (cohorte equivalente). Este artículo explora el tiempo requerido para encontrar esa cohorte pasada (desfase) y las diferencias entre las esperanzas de vida en un determinado año (diferencial) a fin de determinar la translación de la medida de periodo, desde la perspectiva de cohorte. Con la tendencia de aumentos de la longevidad cada vez menores en Brasil, los resultados indican que, tanto para hombres como para mujeres, el diferencial se reduce con el tiempo, al paso que el desfase aumenta. Es decir, con el paso de los años, las estimaciones corrientes de periodo y cohorte tienden a aproximarse, mientras las cohortes equivalentes se hacen más remotas.

Palabras clave: Medidas de longevidad. Translación de la esperanza de vida. Diferencial. Desfase. 